Background: The 3'UTR region plays a crucial role in regulating gene expression at posttranscriptional levels. Any changes in sequence in this region can cause numerous pathologies and can also lead to tumour development. The most common changes reported in in the CDKN2A gene are the $148 \mathrm{Ala} / \mathrm{Thr}$ in exon 2 and $500 \mathrm{C}>\mathrm{G}$ and $540 C>T$ in the 3'UTR region. They are suspected of having a great impact on cancer progression. Since the role of these sequence variants in the Polish population in the development of melanoma has not been confirmed, the importance of 3'UTR polymorphisms in the regulation of gene expression was tested.

Material and methods: First, genetic analysis in a group of 285 melanoma patients was performed and the obtained results were correlated with the clinical course of melanoma. Then vectors carrying 3'UTR sequence variants were prepared and the level expression of the reported gene was measured.

Results: Within this study no correlation between the presence of 148Ala/ Thr polymorphism and cancer in the family was observed. There was a correlation between the presence of this polymorphism and breast cancer and melanoma in the same patient. There was no correlation between $500 \mathrm{C}>\mathrm{G}$ polymorphism and tumour localisation, age of diagnosis, and type of cancer in patients' family, but a correlation between the percentage of patients dying and the $500 \mathrm{C}>\mathrm{G}$ variant was observed.

Conclusion: The results of functional tests indicated that the presence of polymorphism in the 3'UTR region of the CDKN2A gene resulted in changes in the level of reporter gene expression.

Keywords: melanoma, CDKN2A, 3'UTR, polymorphisms.

Contemp Oncol (Pozn) 2015; 19 (4): 276-279 DOI: $10.5114 /$ wo.2015.54227

\section{Analysis of sequence variants in the 3'UTR of CDKN2A gene in melanoma patients}

\author{
Anna Przybyła ${ }^{1}, K^{2}$ atarzyna Lamperska ${ }^{2}$, Andrzej Mackiewicz ${ }^{1}$
}

${ }^{1}$ Department of Cancer Immunology, Chair of Medical Biotechnology, Poznan University of Medical Sciences, Poznan, Poland

${ }^{2}$ Laboratory of Cancer Genetics, Greater Poland Cancer Centre, Poznan, Poland

\section{Introduction}

Cutaneous melanoma has an increasing incidence rate worldwide [1]. The aetiology of melanoma, like other cancers, is heterogeneous and involves environmental factors and genetic predispositions [2]. It has been demonstrated that the inactivation or mutations of certain genes lead to tumour development. One of the best known of these genes is CDKN2A (OMIM*600160). It was identified as the first high-penetrance melanoma susceptibility gene linked with family history of melanoma, young age of onset, and multiple primary tumours [3-5]. The CDKN2A gene is located on chromosome 9p21 and, what is very interesting, encodes two different proteins: p16 and p14ARF, which are involved in cell cycle regulation [6]. In the Polish population, mutations in the CDKN2A gene are very rare $[7,8]$. Thus, more attention has been focused on polymorphisms and non-coding regions of CDKN2A that play a significant role in regulation of gene expression [9].

The messenger RNA 3'-untranslated region (3'UTR) is emerging as critically important in regulating gene expression at posttranscriptional levels. Posttranscriptional events comprise pre-mRNA processing, nucleo-cytoplasmic export, mRNA localisation, mRNA stabilisation, and translational regulation [10]. Abnormalities in any of these important processes can lead to a number of diseases, including the development of cancer [11]. The most commonly reported changes in non-coding regions of the gene CDKN2A, suspected of having an impact on risk [12] and progressive disease [13], are polymorphisms $500 \mathrm{C}>\mathrm{G}$ and $540 \mathrm{C}>\mathrm{T}$ in episode 3'UTR. It is believed that substitutions $540 C>T$ can affect the transport, stability, and translation of p16 mRNA, and consequently, the function of p16 and p14ARF. It is said that there is a correlation between the presence of changes in 3'UTR and a shorter survival time after melanoma remission [14]. In the Polish population the incidence of these two polymorphisms and determination their role in the origin and course of the disease are still unclear.

In these circumstances we decided first of all to perform the genetic analysis of the 3'UTR region of CDKN2A gene in a group of 285 melanoma patients, and to correlate those results with the clinical course of melanoma such as: age of first diagnosis, tumour localisation, survival rate, age of death, coexistence of another type of cancer, and familial history of disease (data published in 2007) [15]. The second step of our experiments was to carry out a functional analysis in vitro in order to assess whether the presence of polymorphisms in the 3'UTR region have any influence on the CDKN2A level expression.

\section{Material and methods}

Blood samples and clinical features of the disease were collected from 285 melanoma patients. 
The study was approved by Regional Bioethics Committee (RBC) in Poznan, Poland and written informed consent was obtained from all melanoma patients and healthy volunteers involved in the study. The patients records was anonymized and de-identified prior to analysis.

DNA isolation. DNA was isolated from whole blood samples or from PBMC stored in liquid nitrogen, using a Wizard Genomic Extraction Kit (Promega, USA) according to the manufacturer's protocol.

PCR-SSCP analysis. The following set of primers for the 3'UTR analysis were used: (1) 5' CCg gTA ggg ACg gCA AgA gA 3', (2) 5' CTg Tag Gac CCT Cgg TgA CTg ATg 3', (3) 5' CTg TAg gAC CTT Cgg TgA CTg ATg 3', (4) 5' gAC ATC CCC gAT TgA AAg AAC 3', (5) 5' TTT ACg gTA gTg ggg gAA gg 3', (6) 5' gTC CTg CCT TTT AAC gTA gA 3', (7) 5' CCT Gag CTT CCC TAg TTC AC 3', (8) 5' CgC CCT AAg CgC ACA TTC AT 3', (9) 5' TTT TgA gTg AAT gAA TgA AAA TT 3'. Primers were labelled on the $5^{\prime}$ end with $\left[\gamma^{32}\right.$ P]ATP $(3000 \mathrm{Ci} / \mathrm{mmol}$, Amersham). The reaction volume for PCR was $5 \mu$ and included $1 \times P C R$ buffer, $1.5 \mathrm{mM} \mathrm{MgCl}, 0.2 \mathrm{mM}$ dNTPs, $1.5 \mu \mathrm{M}$ of appropriate primers, $0.25 \mathrm{U}$ of aTaq DNA polymerase (Promega), and 50 ng of genomic DNA. Standard PCR conditions were applied, with annealing temperature $58^{\circ} \mathrm{C}$ for all primer sets. Electrophoresis was carried out at room temperature at 25 W. Gels were transferred onto Whatman 3 MM paper, dried, and autoradiographed.

Sequencing analysis. Sequencing reactions were performed using the fmol DNA Cycle Sequencing System (Promega) with the same set of primers that were used in the initial PCR amplification. Statistical analysis was performed using Shapiro-Wilk and Lilliefors tests and then Student' $t$-test, $\chi^{2}$ test with Yate's modifications, and Fisher's exact test. Statistical significance of clinical features was tested by Mann-Whitney test.

Cell lines and culture conditions: For functional analysis the melanoma cell lines MICH-2, MeWo, WM35, WM902B, and HSK MEC.1 (human skin microvascular endothelial cells) were used. Cells were maintained in DMEM (Gibco Invitrogen) medium supplemented with 10\% FBS (Gibco Invitrogen) and $80 \mathrm{mg} / \mathrm{l}$ gentamycin (KRKA). Cells were grown at $37^{\circ} \mathrm{C}$ in an atmosphere of $95 \%$ air $-5 \% \mathrm{CO}_{2}$.

Plasmids: To prepare the $500 \mathrm{G} / \mathrm{C}$ and $540 \mathrm{C} / \mathrm{T}$ variants of 3'UTR CDKN2A gene, a pair of complementary $24 \mathrm{nt}$ primers containing the desired mutations was used: (1) 5' ggT CTA Gac ATC CCC gAT TgA AAg AAC 3', (2) 5' CTg ggA TCC TTT TgA gTg AAT gAA Tg 3', (3) 5' TCT gag AAA CCT Cgg gAA ACT T 3', (4) 5' AAg TTT CCC gAg gTT TCT CAg A 3', (5) 5' gTC CTA CAg ggC TAC AAC TgC CC 3', (6) 5' ggg CAg TTg Tag CCC TgT Agg AC 3'. In the next step, products of PCR reactions were cloned into the phRL-CMV vector (Promega) at the Xba I and BamH I sites (Promega).

Luciferase assays in cell lines. A total of $4 \times 10^{5}$ cells were seeded in six-well plates 24 hours before transfection. Cells were transfected at $\sim 90 \%$ confluence using FuGene HD transfection reagents (Roche Diagnostic), according to the manufacturer's instructions. Cells were harvested 24 hours after transfection, and luciferase assays were carried out using reagents of the Dual Luciferase Reporter Assays System (Promega). The presented results of measurements are the average of three independent repeats of each experiment. Statistical analysis was performed using GraphPad InStat v.3.05 (GraphPad Software). The association of genotype and luciferase activity was evaluated by one-way ANOVA test and post-hoc Student-Newman-Keuls test.

\section{Results}

Analysis of clinical data

Analysis of the clinical data in the group of 285 patients was performed and presented in the following publication: Lamperska K., et al. 2007 [15].

\section{PCR-SSCP analysis}

Analysis showed the 500 C/G variant in 62 cases and 148 Ala/Thr polymorphism in seven cases. Both changes together were present in five cases, and the correlation between the variants $500 \mathrm{C} / \mathrm{G}$ and $148 \mathrm{Ala} / \mathrm{Thr}$ was statistically significant ( $p=0.0066)$. Patients with multiple cancers including melanoma did not show polymorphisms in the CDKN2a gene. The presence of mutations in the BRCAl gene, characteristic for the Polish population in all patients with breast cancer as the second, concomitant with melanoma cancer, were investigated [16]. No changes in BRCA1 were found. Polymorphism $500 C / G$ was found in nine patients having cancers in the family, but no correlation with the type of cancer was established. $148 \mathrm{Ala} / \mathrm{Thr}$ was present in only one patient with a family cancer history, together with the 500 C/G variant. Since 148 Ala/Thr was recognised mostly in DNA from patients with no cancer history in the family, correlation analysis between the variant and the type of cancer in the family was not performed. Polymorphism $500 \mathrm{C} / \mathrm{G}$ was found in 26 living persons and 37 dead. The correlation between the percentage of dead patients and the $500 \mathrm{C} / \mathrm{G}$ polymorphism was found to be statistically significant ( $p=0.0252$ ) (Table 1). No correlation was observed for $148 \mathrm{Ala} / \mathrm{Thr}(p=0.5608)$ alone, or for the $500 \mathrm{C} / \mathrm{G}$ plus $148 \mathrm{Ala} / \mathrm{Thr}$ together $(p=0.0509)$. The median age of diagnosis of all the patients was 47 years, while for the carriers of the $500 \mathrm{C} / \mathrm{G}$ variant it was 50 years. No correlation between the age of diagnosis and the presence of the $500 \mathrm{C} / \mathrm{G}$ variant was found $(p=0.5984)$. No correlation was found for carriers of the $148 \mathrm{Ala} / \mathrm{Thr}$ polymorphism and the age of melanoma diagnosis $(p=0.2358)$.

\section{Functional analysis}

Three vectors carrying the three most common sequence variants $(500 \mathrm{C}>\mathrm{G}, 540 \mathrm{C}>\mathrm{T}$, and variant without mutation wild type) together with luciferase reporter gene were constructed and used for tumour cell transfections. In all performed experiments differences in reporter gene expression

Table 1. CDKN2A polymorphisms and number of death and survivals

\begin{tabular}{|c|c|c|c|}
\hline Variable & 0 (death) & 1 (survival) & P (value) \\
\hline \multirow[t]{2}{*}{$500 C>G$} & 37 & 26 & \multirow{2}{*}{$\begin{array}{c}\chi^{2} p=0.0252^{\mathrm{a}} \\
\text { Fisher } p=0.0181^{\mathrm{a}}\end{array}$} \\
\hline & 95 & 127 & \\
\hline \multirow{2}{*}{$\begin{array}{l}500 C>G+148 \\
\text { Ala/Thr }\end{array}$} & 37 & 28 & \multirow{2}{*}{$\begin{array}{c}\chi^{2} p=0.0509^{\mathrm{a}} \\
\text { Fisher } p=0.0352^{\mathrm{a}}\end{array}$} \\
\hline & 95 & 125 & \\
\hline
\end{tabular}


depending on the 3'UTR variant were observed. In our experiments the value of the luminescence emitted by cells transfected with the 3'UTR-wt variant was equated to 1. In HSK MEC.1, WM 902B, WM 35, and MeWo cells 3'UTR-500C >G and 3'UTR-540C $>T$ variants led to a reduction in reporter activity. In the HSK MEC.1 cell line, variant 3'UTR-500C> G, a 2.9-times lower expression of the reporter protein in comparison to the variant 3'UTR-wt $(p=0.0149)$ and a 10-times lower expression of the variant 3'UTR-540C $>$ T $(p=0.0006)$ was observed. These differences were statistically significant.

The WM 902B showed 2.2-times lower activity of luciferase for variant 3'UTR-500C> G compared to the 3'UTR-wt $(p=0.1690)$ and 8.6-times lower for variant 3'UTR-540C> T $(p=0.0013)$, and the difference was statistically significant.

In WM 35 cells variant 3'UTR-500C> G shown 1.3-times lower reporter activity in relation to the 3'UTR-wt $(p=$ $=0.1690$ ) and 4000-times lower active for variant 3'UTR-540C> $\mathrm{T}(p=0.0001)$. This difference was statistically significant.

In another melanoma cell line, MeWo, expression of reporter gene in case of 3'UTR-500C > G and 3'UTR-540C >T was 2.1- and 18.3-times lower than the wild type variant ( $p=0.1850$ and $p=0.0014$, respectively).

Interestingly, in $\mathrm{MICH}-2$ melanoma cell line variants 3'UTR-500C $>$ G and 3'UTR-540C $>$ T showed over 2-times higher luciferase activity than the wild type in this cells ( $p=03499$ and $p=0.3854$, respectively).
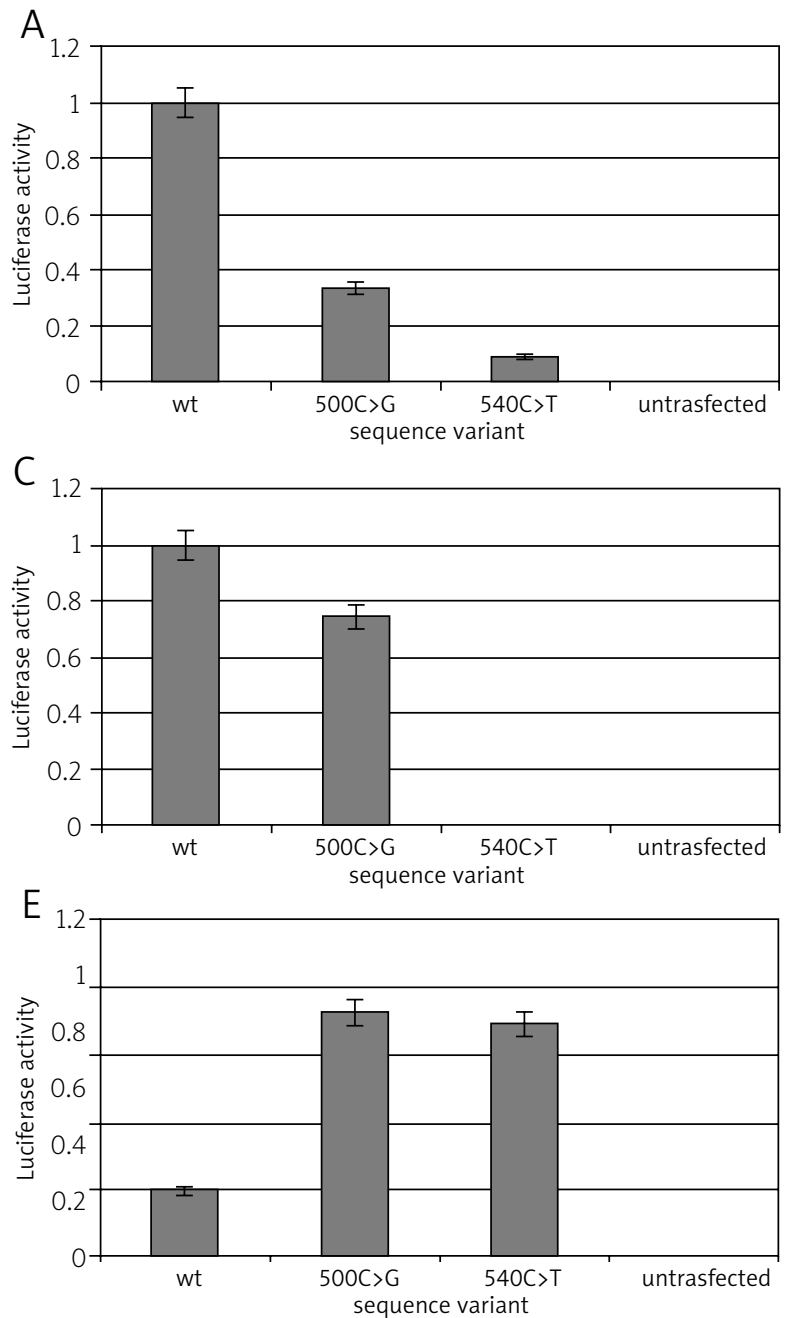

We noticed that in all melanoma cell lines the level of reported gene expression was different in the case of sequence variants relative to the wild type.

\section{Discussion}

The CDKN2A gene is one of the first genes thoroughly analysed in the last decade, due to the high (90\%) percentage of mutations found in melanoma cell lines [17]. Somatic mutations are found in varying proportion of cases and on both sporadic [18] or hereditary melanoma [19]. Most mutations associated with the disease occur in exon $1 \alpha$ and exon 2, affecting the p16 protein [20]. In the Polish population hereditary mutations in the CDKN2A gene are very rare [7], and the polymorphisms are detected with higher incidence in melanoma patients compared to healthy volunteers,

The most common polymorphism recorded in the coding region of CDKN2A is 148Ala/Thr in exon 2, which is significantly more frequent in patients with melanoma than in controls [21]. It was initially described as a common polymorphism, without major effects on p16 function [22]. However, additional reports have implicated CDKN2A 148Ala/Thr as a melanoma susceptibility variant among MPM patients. In Poland this polymorphism was associated with melanoma risk (regardless of family history) [7]. Within our study no correlation between the presence of 148Ala/Thr polymor-
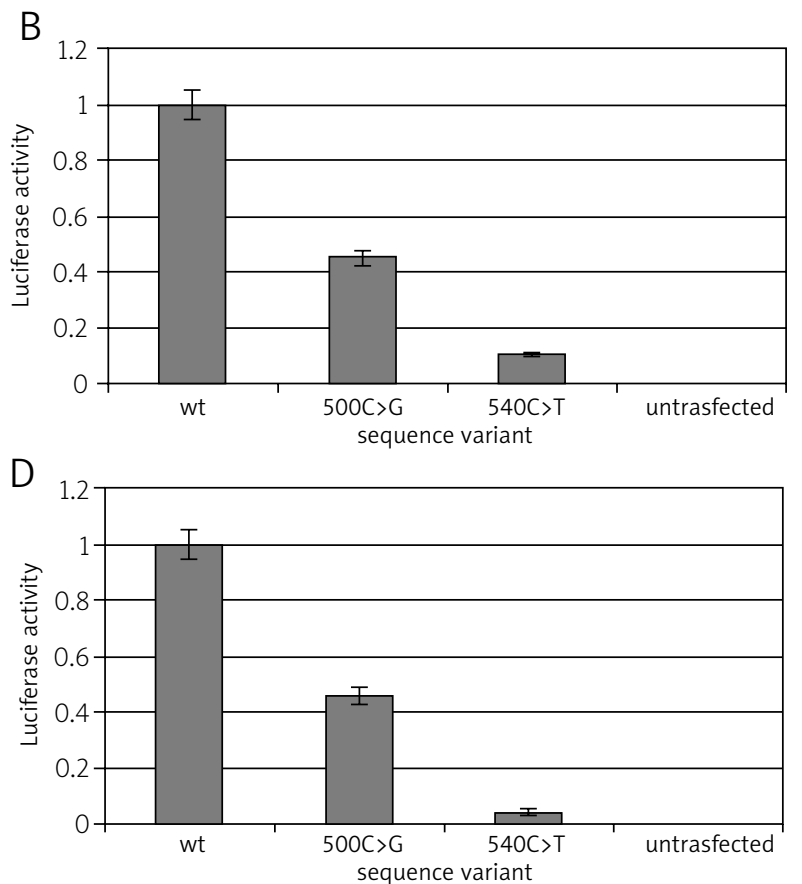

Fig. 1. Impact of the CDKN2A 3'UTR sequence variant on luciferase activity in melanoma cell lines: (A) HSK MEC.1 (human skin microvascular endothelial cells), (B) WM 902B, (C) WM 35, (D) MeWo, and (E) Mich-2 
phism and cancer in the family was observed. Up to now, no familial pathogenic mutations in the CDKN2A gene have been found in Poland. Thus, more attention has been focused on sequence changes and non-coding regions. There are two, often described sequence changes located in the 5 'untranslated region with a proven effect on the p16 protein and, consequently, on the development of melanoma $[23,24]$. The most common sequence changes in 3'UTR of the CDKN2A gene related with melanoma are $500 \mathrm{C}>\mathrm{G}$ and $540 C>T$ [12]. It is said that the both of these changes are correlated with higher risk of melanoma in FMM families [25]. In the Polish population the presence of $500 \mathrm{C}>\mathrm{G}$ and $540 \mathrm{C}>\mathrm{T}$ and their impact on the development of melanoma remains unknown. In the analysed group of patients we found no $540 C>T$ polymorphism. Unfortunately, in our experiments we did not answer the question of how exactly sequence variant $500 \mathrm{C}>\mathrm{G}$ affects melanoma, but the results made it possible to draw a number of conclusions. No relationship was found between the occurrence of polymorphism 500C $>\mathrm{G}$ and the location of the primary tumour, the age at onset, and the type of cancer present in the patient's family. The $500 \mathrm{C}>\mathrm{G}$ polymorphism is correlated with 148Ala/Thr. We found a correlation between the presence of polymorphism 500C $>\mathrm{G}$ and the percentage of deaths, but due to an overly heterogeneous study group, analysis of the overall survival among carriers of this polymorphism was not possible. Still thus compound of the change on the clinical course of melanoma is discussed.

It is well known that the 3'UTR region plays a very important role in cell activity. We decided to conduct a functional in vitro analysis and investigate if the $500 \mathrm{C}>\mathrm{G}$ and $540 C>T$ sequence variants lead to changes in expression level of luciferase reporter gene. Similar analyses were performed by Italian researchers [24]; however, they concerned the 5'UTR. They confirm that the analysed sequence variants ( $c-34 \mathrm{G}>\mathrm{T}$ and $-21 \mathrm{C}>\mathrm{T}$ ) could be a novel loci for susceptibility to melanoma.

In all experiments performed in our laboratory, differences in reporter gene expression depending on 3'UTR variant were observed. This result confirmed the impact of the 3'UTR polymorphisms (500C $>G$ and $540 C>T$ ) in the CDKN2A gene on the level of reporter gene activity, thus they may modulate the expression of the CDKN2A gene in melanoma patients.

The project was supported by grant N N401 063637 funded by the Ministry of Science and Higher Education The authors declare no conflict of interest.

\section{References}

1. Usher-Smith JA, Emery J, Kassianos AP, Walter FM. Risk prediction models for melanoma: a systematic review. Cancer Epidemiol Biomarkers Prev 2014; 23: 1450-63.

2. Jhappan Ch, Noonan FP, Merlino G. Ultraviolet radiation and cutaneous malignant melanoma. Oncogene 2003; 22: 3099-112.

3. Goldstein AM, Struewing JP, Fraser MC, et al. Prospective risk of cancer in CDKN2A germline mutations carriers. J Med Genet 2004; 41:421-4.

4. Gruber F, Kaštelan M, Brajac I, Saftić M, Peharda V, Čabrijan L, Žgombić ZS, Simonić E. Molecular and genetic mechanisms in melano ma. Coll Antropol 2008; 32 Suppl. 2: 147-52.
5. Puig S, Malvehy J, Badenas C, et al. Role of CDKN2A locus in patients with multiple primary melanoma. J Clin Oncol 2005; 23: 3043-51.

6. Nelson AA, Tsao H. Melanoma and genetics. Clinics in Dermatology 2009; 27: 46-52.

7. Dębniak T, Scott RJ, Huzarski T, et al. CDKN2A common variants and their association with melanoma risk: a population-based study. Cancer Res 2005; 65: 835-9.

8. Lamperska KM, Karczewska A, Kwiatkowska E, Mackiewicz A. Analysis of mutations in p16 CDKN2A gene in sporadic and familial melanoma in the polish population. Act Biochim Pol 2002; 49: 369-76.

9. Chatterjee S, Pal JK. Role of 5'- and 3'- untranslated regions of mRNAs in human diseases. Biol Cell 2009; 101: 251-62.

10. Lopez de Silanes I, Paz Quesada M, Esteller M. Aberrant regulation of messenger RNA 3'-untranslated region in human cancer. Cellular Oncology 2007; 29: 1-17.

11. Venables JP. Unbalanced alternative splicing and its significance in cancer. Bioessays 2006; 28: 378-86.

12. Kumar R, Smeds J, Berggren P, Straume O, Rozell BL, Akslen LA, Hemminiki K. A single nucleotide polymorphism in the 3' untranslated region of the CDKN2A gene is common in sporadic primery melanomas but mutations in the CDKN2B, CDKN2C, CDK4 and p53 genes are rare. Int J Cancer 2001; 95: 388-93.

13. Straume O, Smeds J, Kumar R, Hemminki K, Akslen LA. Significant impact of promoter hypermethylation and the $50 \mathrm{C}>\mathrm{T}$ polymorphism of CDKN2A in cutaneous melanoma of the vertical growth phase. Am J Pathol 2002; 161: 229-37.

14. Sauroja I, Smeds J, Vlaykova T, et al. Analysis of G1/S checkpoint regulators in metastatic melanoma. Genes Chromosomes Cancer 2000; 28: 404-14.

15. Lamperska KM, Przybyła A, Kycler W, Mackiewicz A. The CDKN2a common variants: $148 \mathrm{Ala} / \mathrm{Thr}$ and 500C/G in 3'UTR and their association with clinical course of melanoma. Acta Biochim Pol 2007; 54: 119-24.

16. Górski B, Byrski T, Huzarski T, et al. Founder mutations in BRCA1 gene in polish families with breast -ovarian cancer. Am J Hum Genet 2000; 66: 1963-8.

17. Gray-Schopfer VC, da Rocha Dias S, Marais R. The role of B-RAF in melanoma. Cancer Metastasis Rev 2005; 24: 165-83

18. Cachia AR, Indsto JO, McLaren KM, Mann GJ, Arends MJ. CDKN2A mutation and deletion status in thin and thick primary melanoma. Clin Cancer Res 2000; 6: 3511-5.

19. Hussussian JP, Struewing AM, Goldstein PA, et al. Germline p16 mutations in familial melanoma. Nat Genet 1994; 8: 15-21.

20. Meyle KD, Guldberg P. Genetic risk factors for melanoma. Hum Genet 2009; 126: 499-510.

21. Bakos RM, Besch R, Zoratto GG, et al. The CDKN2A p.A148T variant is associated with cutaneous melanoma in Southern Brazil. Exp Dermatol 2011; 20: 890-3.

22. Honrado E, Benítez J, Palacios J. Histopathology of BRCA1 and BRCA2 associated breast cancer. Crit Rev Oncol Hematol 2006; 59 : 27-39.

23. Liu L, Dilworth D, Gao L, Monzon J, Summers A, Lassam N, Hogg D. Mutation in the CDKN2A 5'UTR creates an aberrant initiation codon and predisposes to melanoma. Nat Genet 1999; 21: 128-132

24. Bisio A, Nasti S, Jordan JJ, et al. Functional analysis of CDKN2A/ p16INK4A 5'UTR variants predisposing to melanoma. Hum Mol Genet 2010; 8: 1479-91.

25. Aitken J, Welch J, Duffy D, Milligan A, Green A, Martin N, Hayward N. CDKN2A variants in a population-base sample of Queensland families with melanoma. J Natl Cancer Inst 1999; 91: 446-52.

\section{Address for correspondence}

\section{Anna Przybyła}

Department of Cancer Immunology

Chair of Medical Biotechnology

Poznan University of Medical Sciences

Poznan, Poland

e-mail: przybka@wp.pl

Submitted: 7.06.2015

Accepted: $\quad 20.08 .2015$ 